\title{
REPRESENTATIONS OF SEMIGROUPS AND THE TRANSLATIONAL HULL OF A REGULAR REES MATRIX SEMIGROUP
}

\author{
BY \\ MARIO PETRICH
}

1. Introduction. The translational hull $\Omega(S)$ of a weakly reductive semigroup $S$ plays an important role in the construction of ideal extensions of $S$ (4.4 of [3], [6]), and contains an isomorphic copy of $S$ as a densely embedded ideal [5], [6]. The purpose of this paper is to show that a great number of representations of semigroups are essentially homomorphisms into the translational hull $\Omega(S)$ of a suitable regular Rees matrix semigroup $S=\mathscr{M}^{0}(G ; I, \Lambda ; P)$. In fact, a number of important semigroups are isomorphic to $\Omega(S)$ for some such $S$. For example, let 1 be the one element group, $I$ be a nonempty set, $\mathfrak{P}(I)$ be the set of nonempty subsets of $I$. Then we have

$$
\begin{aligned}
\Omega\left(\mathscr{M}^{0}(1 ; I, I ; \Delta)\right) & \cong V(I), \\
\Omega\left(\mathscr{M}^{0}(1 ; I, \mathfrak{P}(I) ; P)\right) & \cong W(I) \quad \text { with } p_{A x}=1 \text { if } x \in A, \text { and } 0 \text { otherwise, } \\
\Omega\left(\mathscr{M}^{0}(1 ; \mathfrak{B}(I), \mathfrak{P}(I) ; P)\right) & \cong \mathscr{B}(I) \quad \text { with } p_{A B}=1 \text { if } A \cap B \neq \square, \text { and } 0 \text { otherwise, }
\end{aligned}
$$

where $V(I), W(I), \mathscr{B}(I)$ are the semigroups of all partial one-to-one transformations, partial transformations (written on the left), binary relations on $I$, respectively (see [10] and the references listed there). It is easy to see that for $S$ a right zero semigroup, $\Omega(S) \cong \mathscr{T}_{S}$, the semigroup of all transformations on $S$ written on the right [3, Exercise 8 for $\S 1.3]$. For $S=\mathscr{M}^{0}\left(\mathfrak{M} F ; I_{A}, \Lambda_{A} ;\left[e_{\kappa} f_{i}\right]\right)$, with the notation of $[4, \S 2], \Omega(S)$ is isomorphic to the semigroup of all endomorphisms of the vector space $A$ over the division ring $F$ [4], [11].

We will show that the Schützenberger representation associated with a regular $\mathscr{D}$-class and some other representations are closely related to homomorphisms into either the translational hull or the semigroup of all right translations of a regular Rees matrix semigroup. $\$ 2$ has an auxiliary character; in it we introduce the needed notation, the trace of a semigroup and the right wreath product, and discuss the constructions to be used later. In $\$ 3$ we consider the trace of a regular $\mathscr{D}$-class, a homomorphism into its translational hull, and connections with the Schützenberger representations and the sum thereof. At the end, we find several embeddings of a regular semigroup. In $\S 4$ we introduce the fragment of a regular $\mathscr{D}$-class and of a regular semigroup, perform an analysis similar to that in $\S 3$, and

Received by the editors October 18, 1968 and, in revised form, January 15, 1969. 
establish the connection with the results in that section and with transitive representations by partial transformations. We introduce the contour of a semigroup in $\$ 5$, discuss its connection with the embedding of an inverse semigroup into a symmetric inverse semigroup, and illustrate the previous constructions on the example of a bisimple $\omega$-semigroup. Finally, in $\$ 6$, we consider a representation induced by a semilattice congruence on the semigroup and its connection with some known representations.

2. Notation and preliminaries. We generally follow the terminology used in [3]; the notation is that introduced in [9] while undefined symbols have the meaning given to them in [3] (thus our notation sometimes differs from that in [3]). Multiplication is usually denoted by juxtaposition. The zero of any semigroup is denoted by 0 and the identity by 1 . The functions are written either on the left or on the right (as convenient).

Let $S$ be any semigroup. The left [right] translations of $S$ are written as operators on the left [right]; $\Lambda(S)[\mathrm{P}(S)]$ denotes the semigroup of all left [right] translations of $S$ under multiplication $\left(\lambda \lambda^{\prime}\right) x=\lambda\left(\lambda^{\prime} x\right)\left[x\left(\rho \rho^{\prime}\right)=(x \rho) \rho^{\prime}\right]$ for all $x \in S$. The translations $\lambda \in \Lambda(S), \rho \in \mathrm{P}(S)$ are linked if $x(\lambda y)=(x \rho) y$ for all $x, y \in S$. The subsemigroup of the direct product $\Lambda(S) \times \mathrm{P}(S)$ consisting of all pairs of linked left and right translations is the translational hull $\Omega(S)$ of $S$.

Let $K$ be a nonempty set. A function $\gamma$ on a subset of $K$ (denoted $d \gamma$ ) onto a subset of $K$ (denoted $\boldsymbol{r} \gamma$ ) is a partial transformation on $K$ (the empty transformation 0 satisfies $d 0=r 0=\square$ ). The set of all partial transformations on $K$ written as operators on the left [right] under the multiplication:

$$
\begin{gathered}
d\left(\gamma \gamma^{\prime}\right)=\left\{x \in K \mid x \in d \gamma^{\prime}, \gamma^{\prime} x \in d \gamma\right\}, \\
\left(\gamma \gamma^{\prime}\right) x=\gamma\left(\gamma^{\prime} x\right) \quad \text { if } x \in d\left(\gamma \gamma^{\prime}\right) \\
{\left[d\left(\gamma \gamma^{\prime}\right)=\left\{x \in K \mid x \in d \gamma, x \gamma \in d \gamma^{\prime}\right\}, x\left(\gamma \gamma^{\prime}\right)=(x \gamma) \gamma^{\prime} \text { if } x \in d\left(\gamma \gamma^{\prime}\right)\right]}
\end{gathered}
$$

is a semigroup denoted by $W(K)\left[W^{\prime}(K)\right]$.

The following results are taken from the author's paper [9]. Let $K$ be a nonempty set and $G$ be a group. If $D$ is a nonempty subset of $K$ and $\psi: D \rightarrow G$ is a function, we write $d \psi=D$. If $\psi^{\prime}$ is another such function, and $d \psi \cap d \psi^{\prime} \neq \square$, define $\psi \cdot \psi^{\prime}$ by:

$$
\boldsymbol{d}\left(\psi \cdot \psi^{\prime}\right)=\boldsymbol{d} \psi \cap \boldsymbol{d} \psi^{\prime}, \quad k\left(\psi \cdot \psi^{\prime}\right)=(k \psi)\left(k \psi^{\prime}\right) \quad \text { if } k \in \boldsymbol{d}\left(\psi \cdot \psi^{\prime}\right) .
$$

If also $\beta \in W^{\prime}(K)$, define ${ }^{\beta} \psi$ by:

$$
\boldsymbol{d}^{\beta} \psi=\{k \in K \mid k \in \boldsymbol{d} \beta, k \beta \in \boldsymbol{d} \psi\}, \quad k^{\beta} \psi=k \beta \psi \quad \text { if } k \in \boldsymbol{d}^{\beta} \psi,
$$

if $d^{\beta} \psi \neq \square$. Now let $T$ be a subsemigroup of $W^{\prime}(K)$ containing the empty transformation 0 . Let $G$ wr $T$ denote the set

$$
\{(\psi, \beta) \mid 0 \neq \beta \in T, \psi: d \beta \rightarrow G\} \cup 0
$$

together with the multiplication

$$
(\psi, \beta)\left(\psi^{\prime}, \beta^{\prime}\right)=\left(\psi^{\beta} \psi^{\prime}, \beta \beta^{\prime}\right) \text { if } \beta \beta^{\prime} \neq 0 \text {, and } 0 \text { otherwise, }
$$


and 0 acts as the zero. Then $G$ wr $T$ is a semigroup; we call it the right wreath product of $G$ and $T$ (more precisely, the notation should include the set $K$; in [9], the notation $R(M, G)$ is used for $G$ wr $\left.W^{\prime}(M)\right)$.

Next let $S=\mathscr{M}^{0}(G ; I, \Lambda ; P)$ be a regular Rees matrix semigroup. The function $b$ defined by:

$$
\rho \boldsymbol{b}=(\psi, \beta) \quad(\rho \in \mathrm{P}(S)), \quad 0 \boldsymbol{b}=0
$$

where $(1 ; i, \mu) \rho=(\mu \psi ; i, \mu \beta)$ if $(1 ; i, \mu) \rho \neq 0$, is an isomorphism of $\mathrm{P}(S)$ onto $G$ wr $W^{\prime}(\Lambda)$.

By $M^{\prime}(G, \Lambda)$ denote the semigroup of all row-monomial $\Lambda \times \Lambda$ matrices over $G^{0}$ (row by column multiplication). The function $d$ defined by:

$$
(\psi, \beta) d=\left(b_{\mu \nu}\right) \quad\left((\psi, \beta) \in G \text { wr } W^{\prime}(\Lambda)\right),
$$

where $\left(b_{\mu v}\right)$ is the $\Lambda \times \Lambda$ matrix with

$$
b_{\mu \nu}=\mu \psi \text { if } \mu \in d \beta, \mu \beta=\nu, \text { and } 0 \text { otherwise, }
$$

and $0 d=0$ is the $\Lambda \times \Lambda$ matrix all of whose entries are zero, is an isomorphism of $G$ wr $W^{\prime}(\Lambda)$ onto $M^{\prime}(G, \Lambda)$ (this is also noted on p. 85 of [1]).

One defines analogously the left wreath product; the semigroups $\Lambda(S), W(I)$ wl $G$, and $M(I, G)$ are isomorphic (using the obvious notation).

The projection $\pi_{\mathrm{P}}:(\lambda, \rho) \rightarrow \rho$ of $\Omega(S)$ into $\mathrm{P}(S)$ is always a homomorphism, and for $S$ as above, it is one-to-one if and only if $S$ is left reductive. If $G=1$, the one element group, we identify $1 \mathrm{wr} W^{\prime}(M)$ with $W^{\prime}(M)$. To simplify the notation, we will use $\pi_{\mathrm{P}}, \boldsymbol{b}, \boldsymbol{d}$ for different semigroups $S$.

Let $A$ be an index set and for every $\alpha \in A$, let $S_{\alpha}$ be a semigroup with zero $0_{\alpha}$ and at least one more element, and suppose that $S_{\alpha} \cap S_{\beta}=\square$ if $\alpha \neq \beta$. Let 0 be any element not contained in any $S_{\alpha}$, and let $S=\left[\bigcup_{\alpha \in A}\left(S_{\alpha} \mid 0_{\alpha}\right)\right] \cup 0$ with the multiplication: if $x, y \in S_{\alpha} \mid 0$ and $x y \neq 0_{\alpha}$ in $S_{\alpha}$, then $x * y=x y$, in all other cases $x * y=0$. Then $S$ is a semigroup; if $T$ is any semigroup isomorphic to $S$ we say that $T$ is an orthogonal sum of semigroups $S_{\alpha}$ and write $T=\sum_{\alpha \in A} \oplus S_{\alpha}$. If $A=\{1,2\}$ we write $T=S_{1} \oplus S_{2}$.

Now let $S$ be any semigroup, $T$ be a union of some of its $\mathscr{D}$-classes, and let 0 be any element not in $S$. Let $\operatorname{Tr}(T)$ be the set $T \cup 0$ together with the multiplication

$$
a * b=a b \quad \text { if } a b \in R_{a} \cap L_{b} \text {, and } 0 \text { otherwise, }
$$

and 0 acts as the zero. By [8, Lemma 4], $\operatorname{Tr}(T)$ is a semigroup; we call it the trace of $T$. When $T$ is a single $\mathscr{D}$-class, this definition agrees with the definition of the trace of a $\mathscr{D}$-class [3, p. 92]. Let $T$ be as above and let $D_{\alpha}, \alpha \in A$, be the $\mathscr{D}$-classes of $S$ contained in $T$. From the proof of [8, Lemma 4], it follows that $\operatorname{Tr}(T)=$ $\sum_{\alpha \in A} \oplus \operatorname{Tr}\left(D_{\alpha}\right)$. If $D_{\alpha}$ is a regular $\mathscr{D}$-class, $\operatorname{Tr}\left(D_{\alpha}\right)$ is completely 0 -simple [8, Theorem 6], otherwise $\left[\operatorname{Tr}\left(D_{\alpha}\right)\right]^{2}=0$. Letting $B$ be the index of regular $\mathscr{D}$-classes in $T, B \subseteq A$, we obtain

$$
\operatorname{Tr}(T)=\sum_{\alpha \in B} \oplus \operatorname{Tr}\left(D_{\alpha}\right) \oplus Q
$$


if $\square \neq B \neq A, \operatorname{Tr}(T)=Q$ if $B=\square$, where $Q=\left(\bigcup_{\alpha \in A \backslash B} D_{\alpha}\right) \cup 0$ with zero multiplication, and otherwise

$$
\operatorname{Tr}(T)=\sum_{\alpha \in A} \oplus \operatorname{Tr}\left(D_{\alpha}\right) .
$$

The direct product of a family of semigroups $S_{\alpha}, \alpha \in A$, is denoted by $\prod_{\alpha \in A} S_{\alpha}$.

When $S$ is an inverse semigroup, the "adjoining multiplication" on the set $S$ defined on p. 310 of [14], agrees with the partial operation on $\operatorname{Tr}(S) \mid 0$ obtained by removing the zero and declaring $a * b$ undefined if $a b \notin R_{a} \cap L_{b}$.

3. The trace of a regular $\mathscr{D}$-class. Fix a semigroup $S$ and a regular $\mathscr{D}$-class $D$ of $S$. Let $\left\{R_{i} \mid i \in I\right\}$ be the set of $\mathscr{R}$-classes of $S$ contained in $D,\left\{L_{\mu} \mid \mu \in \Lambda\right\}$ be the set of $\mathscr{L}$-classes of $S$ contained in $D$, and write $H_{i \mu}=R_{i} \cap L_{\mu}$. We suppose that $1 \in I \cap \Lambda$ and that $H_{11}=R_{1} \cap L_{1}$ is a group with identity $e$. For every $i \in I$, fix an element $r_{i} \in H_{i 1}$, and for every $\mu \in \Lambda$, fix an element $q_{\mu} \in H_{1 \mu}$. Let

$$
T_{D}=\mathscr{M}^{0}\left(H_{11} ; I, \Lambda ; P\right)
$$

where $P$ is a $\Lambda \times I$ matrix over $H_{11}^{0}$ with $p_{\mu i}=q_{\mu} r_{i}$ if $q_{\mu} r_{i} \in H_{11}$, and 0 otherwise.

Then $P$ is a regular matrix and $T_{D} \cong \operatorname{Tr}(D)$ ([8, Theorem 6], see also p. 92 of [3]), so that $T_{D}$ is, up to an isomorphism, independent of the choice of $H_{11}, r_{i}, q_{\mu}$. For each $i \in I$, fix an idempotent $e_{i} \in R_{i}$ and let $r_{i}^{\prime}$ be the unique inverse of $r_{i}$ in $L_{1}$ such that $r_{i} r_{i}^{\prime}=e_{i}$; for every $\mu \in \Lambda$, fix an idempotent $f_{\mu} \in L_{\mu}$ and let $q_{\mu}^{\prime}$ be the unique inverse of $q_{\mu}$ in $R_{1}$ such that $q_{\mu}^{\prime} q_{\mu}=f_{\mu}$ (note that $r_{i}^{\prime} r_{i}=e=q_{\mu} q_{\mu}^{\prime}$, see [3, p. 93]). For every $s \in S$, define $\lambda^{s}$ and $\rho^{s}$ on $T_{D}$ by

$$
\begin{aligned}
& \lambda^{s}(a ; i, \mu)=\left(r_{j}^{\prime} s r_{i} a ; j, \mu\right) \quad \text { if } s H_{i 1}=H_{j 1}, \text { and } 0 \text { otherwise, } \\
& (a ; i, \mu) \rho^{s}=\left(a q_{\mu} s q_{v}^{\prime} ; i, \nu\right) \quad \text { if } H_{1 \mu} s=H_{1 v}, \text { and } 0 \text { otherwise, } \\
& \lambda^{s} 0=0 \rho^{s}=0,
\end{aligned}
$$

and let

$$
\chi_{D}: s \rightarrow\left(\lambda^{s}, \rho^{s}\right) \quad(s \in S) .
$$

The next theorem is of basic importance for the results of this and the next sections.

THEOREM 1. $\chi_{D}$ is a homomorphism of $S$ into $\Omega\left(T_{D}\right)$.

Proof. First note that if $s H_{i 1}=H_{j 1}$, then $s r_{i} \in H_{j 1}$ so that $r_{j}^{\prime} s r_{i} \in H_{11}$; similarly, if $H_{1 \mu} s=H_{1 v}$, then $q_{\mu} s q_{v}^{\prime} \in H_{11}$. Consequently, both $\lambda^{s}$ and $\rho^{s}$ map $T_{D}$ into itself. Let $s \in S$ and $(a ; i, \mu),(b ; k, \theta) \in T_{D}$. If $q_{\mu} r_{k} \in H_{11}$ and $s H_{i 1}=H_{j 1}$, then

$$
\begin{aligned}
\lambda^{s}[(a ; i, \mu)(b ; k, \theta)] & =\lambda^{s}\left(a q_{\mu} r_{k} b ; i, \theta\right)=\left(r_{j}^{\prime} s r_{i} a q_{\mu} r_{k} b ; j, \theta\right) \\
& =\left(r_{j}^{\prime} s r_{i} a ; j, \mu\right)(b ; k, \theta)=\left[\lambda^{s}(a ; i, \mu)\right](b, k, \theta) ;
\end{aligned}
$$

if $q_{\mu} r_{k} \notin H_{11}$ or $s H_{i 1} \nsubseteq R_{1}$, then $\lambda^{s}[(a ; i, \mu)(b ; k, \theta)]=0=\left[\lambda^{s}(a ; i, \mu)\right](b ; k, \theta)$. Hence $\lambda^{s}$ is a left translation of $T_{D}$; one verifies similarly that $\rho^{s}$ is a right translation of $T_{D}$. 
Further,

$$
(a ; i, \mu)\left[\lambda^{s}(b ; k, \theta)\right]=(a ; i, \mu)\left(r_{m}^{\prime} s r_{k} b ; m, \theta\right)=\left(a q_{\mu} r_{m} r_{m}^{\prime} s r_{k} b ; i, \theta\right)
$$

if $s H_{k 1}=H_{m 1}$ and $q_{\mu} r_{m} \in H_{11}$, and the left-hand side is 0 otherwise; also

$$
\left[(a ; i, \mu) \rho^{s}\right](b ; k, \theta)=\left(a q_{\mu} s q_{v}^{\prime} ; i, v\right)(b ; k, \theta)=\left(a q_{\mu} s q_{v}^{\prime} q_{v} r_{k} b ; i, \theta\right)
$$

if $H_{1 \mu} s=H_{1 v}$ and $q_{v} r_{k} \in H_{11}$, and the left-hand side is 0 otherwise.

Suppose that $s H_{k 1}=H_{m 1}$ and $q_{\mu} r_{m} \in H_{11}$. Let $h_{m}=s r_{k}$; then $h_{m}=s r_{k} \in s H_{k 1}=H_{m 1}$ so that $q_{\mu} s r_{k}=q_{\mu} h_{m} \in H_{11}$ since $q_{\mu} r_{m} \in H_{11}$. Hence $q_{\mu} s r_{k} \in H_{1 \mu} s r_{k} \cap R_{1}$ so that $H_{1 \mu} s r_{k} \cap R_{1} \neq \square$, and thus by [3, Lemma 3.15(ii)], we have $H_{1 \mu} s \cap R_{1} \neq \square$. By (i) of the same lemma, $H_{1 \mu} s$ is an $\mathscr{H}$-class contained in $R_{1}$, and thus $H_{1 \mu} s=H_{1 v}$. Consequently $q_{\nu} \in H_{1 v}$ implies that $q_{\nu}=t_{\mu} s$ for some $t_{\mu} \in H_{1 \mu}$. Hence

$$
q_{v} r_{k}=\left(t_{\mu} s\right) r_{k}=t_{\mu}\left(s r_{k}\right)=t_{\mu} h_{m} \in H_{11}
$$

since $q_{\mu} r_{m} \in H_{11}$. We have proved that $s H_{k 1}=H_{m 1}$ and $q_{\mu} r_{m} \in H_{11}$ imply $H_{1 \mu} s=H_{1 v}$ and $q_{v} r_{k} \in H_{11}$; the converse implication is verified analogously. It follows that the left-hand sides of (1) and (2) are simultaneously nonzero.

If $s H_{k 1}=H_{m 1}$ and $q_{\mu} r_{m} \in H_{11}$, then taking into account that $e_{m} \mathscr{R} h_{m}$, we obtain

$$
q_{\mu} r_{m} r_{m}^{\prime} s r_{k}=q_{\mu}\left(r_{m} r_{m}^{\prime}\right)\left(s r_{k}\right)=q_{\mu} e_{m} h_{m}=q_{\mu} h_{m}=q_{\mu} s r_{k}
$$

and similarly

$$
q_{u} s q_{v}^{\prime} q_{v} r_{k}=\left(q_{u} s\right)\left(q_{v}^{\prime} q_{v}\right) r_{k}=\left(q_{\mu} s\right) f_{v} r_{k}=q_{u} s r_{k} .
$$

Comparing (1) and (2) with (3) and (4), we see that $\lambda^{s}$ and $\rho^{s}$ are linked. Therefore $\left(\lambda^{s}, \rho^{s}\right) \in \Omega\left(T_{D}\right)$ so that $\chi_{D}$ maps $S$ into $\Omega\left(T_{D}\right)$.

Let $s, t \in S$ and $(a ; i, \mu) \in T_{D}$. Suppose that $t H_{i 1}=H_{j 1}$ and $s H_{j 1}=H_{k 1}$. Then $s t r_{i} \in s H_{j 1}=H_{k 1}$ so that $s t H_{i 1}=H_{k 1}$ by the dual of [3, Lemma 3.15(i)]. Also $r_{j} r_{j}^{\prime}=e_{j}$ and $t r_{i} \in H_{j 1}$ so that $e_{j}\left(t r_{i}\right)=t r_{i}$. We obtain

$$
\begin{aligned}
\lambda^{s} \lambda^{t}(a ; i, \mu) & =\lambda^{s}\left(r_{j}^{\prime} t r_{i} a ; j, \mu\right)=\left(r_{k}^{\prime} s r_{j} r_{j}^{\prime} t r_{i} a ; k, \mu\right) \\
& =\left(r_{k}^{\prime} \operatorname{se}_{j}\left(t r_{i}\right) a ; k, \mu\right)=\left(r^{\prime} s t r_{i} a ; k, \mu\right)=\lambda^{s t}(a ; i, \mu) .
\end{aligned}
$$

In the case that $t H_{i 1} \cap R_{1}=\square$ or $t H_{i 1}=H_{j 1}$ but $s H_{j 1} \cap R_{1}=\square$, we get $\lambda^{s} \lambda^{t}(a ; i, \mu)$ $=0$ and by the dual of [3, Lemma 3.15(ii)], also that $\lambda^{s t}(a ; i, \mu)=0$. Therefore $\lambda^{s} \lambda^{t}(a ; i, \mu)=\lambda^{s t}(a ; i, \mu)$, so that $\lambda^{s} \lambda^{t}=\lambda^{s t}$. One shows similarly that $\rho^{s} \rho^{t}=\rho^{s t}$, which proves that $\chi_{D}$ is a homomorphism.

Since $D$ is a regular $\mathscr{D}$-class and we have chosen $r_{i}, q_{\mu}$ in $D$, we can write the Schützenberger representation $M_{D}$ of $S$ relative to $D$ with matrices over $H_{11}^{0}$ instead of over $\Gamma(H)^{0}$ (we are merely replacing an inner right translation by the element that induces it). We call this representation the modified Schützenberger representation and denote it also by $M_{D}$. Thus $M_{D}: S \rightarrow M^{\prime}\left(H_{11}, \Lambda\right)$ is a representation of $S$ by $\Lambda \times \Lambda$ row monomial matrices over $H_{11}^{0}$, and we have the following result. 
PROPOSITION 1. $\chi_{D} \pi_{\mathrm{P}} b d$ is the modified Schützenberger representation $M_{D}$.

Proof. For any $s \in S$, we have

$$
s \chi_{D} \pi_{\mathrm{P}} \boldsymbol{b d}=\left(\lambda^{s}, \rho^{s}\right) \pi_{\mathrm{P}} \boldsymbol{b d}=\rho^{s} \boldsymbol{b d}=\left(\psi^{s}, \beta^{s}\right) \boldsymbol{d}=\left(b_{\mu v}^{s}\right),
$$

where

$$
\begin{gathered}
d \beta^{s}=\left\{\mu \in \Lambda \mid H_{1 \mu} s \cap R_{1} \neq \square\right\}, \\
\mu \beta^{s}=\nu \text { and } \mu \psi^{s}=q_{\mu} s q_{v}^{\prime} \quad \text { if } H_{1 \mu} s=H_{1 v},
\end{gathered}
$$

so that $b_{\mu \nu}^{s}=q_{\mu} s q_{v}^{\prime}$ if $H_{1 \mu} s=H_{1 v}$, and 0 otherwise. Therefore $M_{D}=\chi_{D} \pi_{\mathrm{P}} b d$ (see [3, p. 111]).

If $\theta: S \rightarrow T$ is a homomorphism, we denote by $\operatorname{ker} \theta$ the congruence on $S$ induced by $\theta$.

COROllaRy 1. $\operatorname{ker}\left(\chi_{D} \pi_{\mathrm{P}}\right)=\{(s, t) \mid$ if $d \in D$ and either $d s \mathscr{R} d$ or $d t \mathscr{R} d$, then $d s=d t\}$.

Proof. Since both $\boldsymbol{b}$ and $\boldsymbol{d}$ are one-to-one, Proposition 1 implies that $\operatorname{ker}\left(\chi_{D} \pi_{\mathrm{P}}\right)$ $=\operatorname{ker} M_{D} ;$ the latter is given in [3, Lemma 3.18].

The "left" versions of Proposition 1 and its corollary also hold and correspond to the dual Schützenberger representation. Denoting by $M_{D}^{\prime}$ the modified dual Schützenberger representation (i.e., writing elements of $H_{11}$ instead of inner left translations), we have another consequence of the above results.

COROllary 2. For every $s \in S, P M_{D}^{\prime}(s)=M_{D}(s) P$.

Proof. This follows easily from Proposition 1 and its dual, the linking of $\lambda^{s}$ and $\rho^{s}$ (Theorem 1), and [9, Theorem 7].

When $S$ is a regular semigroup, to the sum of all direct and dual (or only direct, respectively) Schützenberger representations correspond the following homomorphisms (again the "left" versions will be omitted).

Proposition 2. Let $S$ be a regular semigroup and let $\Delta$ be an index set of its $\mathscr{D}$-classes. Then the mapping $\chi$ defined by:

$$
\chi: s \rightarrow\left(s \chi_{D}\right)_{D \in \Delta} \quad(s \in S),
$$

is an isomorphism of $S$ into $\prod_{D \in \Delta} \Omega\left(T_{D}\right)$. The mapping $\chi^{\mathrm{P}}$ defined by:

$$
\chi^{\mathrm{P}}: s \rightarrow\left(s \chi_{D} \pi_{\mathrm{P}}\right)_{D \in \Delta} \quad(s \in S)
$$

is a homomorphism of $S$ into $\prod_{D \in \Delta} \mathrm{P}\left(T_{D}\right)$ with

$$
\operatorname{ker} \chi^{\mathrm{P}}=\{(s, t) \mid \text { if } x \in S \text { and either } x s \mathscr{R} x \text { or } x t \mathscr{R} x \text {, then } x s=x t\} \text {. }
$$

Moreover, if $S$ is an inverse semigroup, $\chi^{\mathrm{P}}$ is one-to-one.

Proof. This follows easily from Theorem 1, Proposition 1, and Theorems 3.19 and 3.21 of [3]. 
We will next establish that for a regular semigroup $S, \chi$ is related to an isomorphism of $S$ into the translational hull of the trace of $S$. We need an auxiliary result which seems to be of independent interest.

Lemma 1. Let $S_{\alpha}, \alpha \in A$, be a disjoint family of semigroups with zero satisfying $S_{\alpha}^{2}=S_{\alpha}$. Then $\prod_{\alpha \in A} \Omega\left(S_{\alpha}\right) \cong \Omega\left(\sum_{\alpha \in A} \oplus S_{\alpha}\right)$.

Proof. Define $\theta$ by

$$
\theta:\left(\left(\lambda^{\alpha}, \rho^{\alpha}\right)\right)_{\alpha \in A} \rightarrow(\lambda, \rho) \quad\left(\left(\lambda^{\alpha}, \rho^{\alpha}\right) \in \Omega\left(S_{\alpha}\right)\right),
$$

where $\lambda 0=0 \rho=0$ and $\lambda a=\lambda^{\alpha} a, a \rho=a \rho^{\alpha}$ if $a \in S_{\alpha} \mid 0_{\alpha}$. The proof that $\theta$ is an isomorphism of $\prod_{\alpha \in A} \Omega\left(S_{\alpha}\right)$ into $\Omega\left(\sum_{\alpha \in A} \oplus S_{\alpha}\right)$ is straightforward and is omitted (it does not require the restriction $S_{\alpha}^{2}=S_{\alpha}$ ). To prove that $\theta$ is onto, let

$$
(\phi, \psi) \in \Omega\left(\sum_{\alpha \in A} \oplus S_{\alpha}\right)
$$

and $a \in S_{\alpha}$ such that $\phi a \neq 0$. Then $a=x y$ for some $x, y \in S_{\alpha}$, so that $(\phi x) y=\phi(x y)$ $=\phi a \neq 0$. Since $y \in S_{\alpha}$, it follows that $\phi x \in S_{\alpha}$, and hence also $\phi a \in S_{\alpha}$. Consequently $\phi$ maps each $S_{\alpha}$ into itself; similarly $\psi$ maps each $S_{\alpha}$ into itself. Letting $\lambda^{\alpha}=\left.\phi\right|_{S_{\alpha}}$, $\rho^{\alpha}=\left.\psi\right|_{S_{\alpha}}$, we get $\left(\left(\lambda^{\alpha}, \rho^{\alpha}\right)\right)_{\alpha \in A} \theta=(\phi, \psi)$ and hence $\theta$ is onto.

The next theorem provides two embeddings of a regular semigroup.

THEOREM 2. Every regular semigroup can be embedded both in the direct product of the translational hulls of the traces of its different $\mathscr{D}$-classes and in the translational hull of its trace.

Proof. Taking into account that for a regular semigroup $S$ every $\mathscr{D}$-class $D$ is regular and that then $\operatorname{Tr}(D) \cong T_{D}$, the first statement of the theorem follows from the first statement of Proposition 2. We have seen in $\S 2$ that $\operatorname{Tr}(S) \cong \sum_{D \in \Delta} \oplus \operatorname{Tr}(D)$ if $S$ is regular and $\Delta$ is an index set of its $\mathscr{D}$-classes. Hence by Lemma 1 ,

$$
\Omega(\operatorname{Tr}(S)) \cong \prod_{D \in \Delta} \Omega(\operatorname{Tr}(D))
$$

which together with the first statement of the theorem establishes the second.

4. The fragment of a regular $\mathscr{D}$-class. Let $D$ be a regular $\mathscr{D}$-class of a semigroup $S$, and let $L_{1}, R_{1}, e, I, \Lambda$ etc. have the meaning given to them at the beginning of $\S 3$. We define the fragment $F_{D}$ of $D$ by $F_{D}=\mathscr{M}^{0}\left(1 ; L_{1}, R_{1} ; P\right)$ where $P=\left(p_{r l}\right)$ with $p_{r l}=1$ if $r l=e$, and 0 otherwise. Then $P$ is a regular matrix since $D$ is a regular D-class.

Proposition 3. $F_{D}$ is (up to an isomorphism) independent of the choice of the $\mathscr{L}$-class $L_{1}$ and the $\mathscr{R}$-class $R_{1}$ in $D$.

Proof. Let $F_{D}, L_{1}, R_{1}$, and $e$ be as above, let $L_{2}$ and $R_{2}$ be an $\mathscr{L}$ and an $\mathscr{R}$-class in $D$ with an idempotent $f$ in $H_{22}=L_{2} \cap R_{2}$, and let $F_{D}^{\prime}=\mathscr{M}^{0}\left(1 ; L_{2}, R_{2} ; T\right)$, 
$T=\left(t_{r l}\right)$, be the corresponding fragment of $D$. Fix an element $a$ in $R_{1} \cap L_{2}$ and let $a^{\prime}$ be its unique inverse in $R_{2} \cap L_{1}$. Then by [3, Lemma 2.2] (Green's lemma),

$$
\phi: l \rightarrow l a \quad\left(l \in L_{1}\right), \quad \psi: r \rightarrow a^{\prime} r \quad\left(r \in R_{1}\right)
$$

are one-to-one mappings of $L_{1}$ onto $L_{2}$ and of $R_{1}$ onto $R_{2}$, respectively. By [3, Theorem 2.2], the mappings $x \rightarrow a^{\prime} x a$ and $y \rightarrow a y a^{\prime}$ are mutually inverse isomorphisms of $H_{11}$ onto $H_{22}$ and of $H_{22}$ onto $H_{11}$, respectively. Hence, if $p_{r l}=1$, then $r l=e$ so that $(r \psi)(l \phi)=\left(a^{\prime} r\right)(l a)=a^{\prime} e a=f$; conversely, if $t_{r \psi, l \phi}=1$, then $a^{\prime}(r l) a$ $=\left(a^{\prime} r\right)(l a)=(r \psi)(l \phi)=f$ so that $r l=e$. Consequently $p_{r l}=1$ if and only if $t_{r \psi, l \phi}=1$. By [3, Theorem 3.11], it follows that $F_{D} \cong F_{D}^{\prime}$.

Note that the corresponding statement is also true for $T_{D}$ which follows from the fact that for any choice of $H_{11}, r_{i}, q_{\mu}, T_{D}$ is isomorphic to $\operatorname{Tr}(D)$. For every $s \in S$, define $\lambda^{s}$ and $\rho^{s}$ on $F_{D}$ by

$$
\begin{aligned}
& \lambda^{s}(1 ; l, r)=(1 ; s l, r) \quad \text { if } s l \in L_{1}, \text { and } 0 \text { otherwise, } \\
& (1 ; l, r) \rho^{s}=(1 ; l, r s) \quad \text { if } r s \in R_{1}, \text { and } 0 \text { otherwise, } \\
& \lambda^{s} 0=0 \rho^{s}=0,
\end{aligned}
$$

and let

$$
\xi_{D}: s \rightarrow\left(\lambda^{s}, \rho^{s}\right) \quad(s \in S) .
$$

THEOREM 3. $\xi_{D}$ is a homomorphism of $S$ into $\Omega\left(F_{D}\right)$.

Proof. Let $s \in S$ and $(1 ; x, y),(1 ; z, w) \in F_{D}$. If $y z=e$ and $s x \in L_{1}$, then

$$
\begin{aligned}
\lambda^{s}[(1 ; x, y)(1 ; z, w)] & =\lambda^{s}(1 ; x, w)=(1 ; s x, w)=(1 ; s x, y)(1 ; z, w) \\
& =\left[\lambda^{s}(1 ; x, y)\right](1 ; z, w) ;
\end{aligned}
$$

if either $y z \neq e$ or $s x \notin L_{1}$, then $\lambda^{s}[(1 ; x, y)(1 ; z, w)]=0=\left[\lambda^{s}(1 ; x, y)\right](1, z, w)$. Hence $\lambda^{s}$ is a left translation of $F_{D}$; one shows similarly that $\rho^{s}$ is a right translation of $F_{D}$. Further,

$$
(1 ; x, y)\left[\lambda^{s}(1 ; z, w)\right]=(1 ; x, y)(1 ; s z, w)=(1 ; x, w)
$$

if $s z \in L_{1}$ and $y s z=e$, and

$$
\left[(1 ; x, y) \rho^{s}\right](1 ; z, w)=(1 ; x, y s)(1 ; z, w)=(1 ; x, w)
$$

if $y s \in R_{1}$ and $y s z=e$. Since $z \in L_{1}, z=v e$ for some $v \in S^{1}$. Thus, if $y s z=e$, we have $e=y(s z), s z=(s v) e$, that is $s z \mathscr{L} e$ and $s z \in L_{1}$. Hence $y s z=e$ implies $s z \in L_{1}$, and similarly also $y s \in R_{1}$. It then follows from (5) and (6) that $\lambda^{s}[(1 ; x, y)(1 ; z, w)]$ $=\left[\lambda^{s}(1 ; x, y)\right](1 ; z, w)$ if $y s z=e$; if $y s z \neq e$, then both sides are zero. Hence $\lambda^{s}$ and $\rho^{s}$ are linked.

Let $s, t \in S$. If $s t x \in L_{1}$, then $t x \in L_{1}$ and we have

$$
\lambda^{s} \lambda^{t}(1 ; x, y)=\lambda^{s}(1 ; t x, y)=(1 ; s t x, y)=\lambda^{s t}(1 ; x, y),
$$


while if st $\notin L_{1}$, then $\lambda^{s} \lambda^{t}(1 ; x, y)=0=\lambda^{s t}(1 ; x, y)$. Thus $\lambda^{s} \lambda^{t}=\lambda^{s t}$ and similarly $\rho^{s} \rho^{t}=\rho^{s t}$, which proves that $\xi_{D}: s \rightarrow\left(\lambda^{s}, \rho^{s}\right)$ is a homomorphism.

Definition 1. We call the representation $\Phi_{D}: S \rightarrow W^{\prime}\left(R_{1}\right)$, defined by $\Phi_{D}=$ $\xi_{D} \pi_{\mathrm{P}} b$, the Ponizovski representation of $S$ relative to the regular $\mathscr{D}$-class $D$. Explicitly

$$
\Phi_{D}: s \rightarrow \beta^{s} \quad(s \in S)
$$

where $d \beta^{s}=\left\{x \in R_{1} \mid x s \in R_{1}\right\}$ and $x \beta^{s}=x s$ if $x \in d \beta^{s}$.

Proposition 4. $\Phi_{D}$ is (up to equivalence) independent of the choice of the $\mathscr{R}$-class $R_{1}$ in $D$.

Proof. Let $R_{2}$ be another $\mathscr{R}$-class contained in $D$. For any $\mathscr{L}$-class $L_{2}$, we let $a, a^{\prime}$, and $\psi$ be as in the proof of Proposition 3. We also let $s \rightarrow \gamma^{s}$ be the corresponding representation relative to $R_{2}$. We must show that $\left(d \beta^{s}\right) \psi=d \gamma^{s}$ and $\left(x \beta^{s}\right) \psi=(x \psi) \gamma^{s}$ if $x \in d \beta^{s}$. If $y \in\left(d \beta^{s}\right) \psi$, then letting $x=y \psi^{-1}$, we have $x \in d \beta^{s}$ so that $x s \in R_{1}$, and thus $(x s) \psi \in R_{2}$. But $(x s) \psi=a^{\prime}(x s)=\left(a^{\prime} x\right) s=(x \psi) s$ and hence $y, y s \in R_{2}$ which implies that $y \in d \gamma^{s}$. Consequently $\left(d \beta^{s}\right) \psi \subseteq d \gamma^{s}$; a similar argument shows that $\boldsymbol{d} \gamma^{s} \subseteq\left(d \beta^{s}\right) \psi$, so $\left(d \beta^{s}\right) \psi=d \gamma^{s}$. If $x \in d \beta^{s}$, then

$$
\left(x \beta^{s}\right) \psi=a^{\prime}(x s)=\left(a^{\prime} x\right) s=(x \psi) \gamma^{s},
$$

which then establishes the equivalence of the two representations.

The next result shows the connection between the Schützenberger and the Ponizovski representations. We note that the Ponizovski representation and its connection with the Schützenberger representation were found independently by E. J. Tully, Jr. [15]. Define $\zeta$ by

$$
\zeta:\left(a_{\mu v}\right) \rightarrow\left(c_{x y}\right) \quad\left(\left(a_{\mu v}\right) \in M^{\prime}\left(H_{11}, \Lambda\right)\right)
$$

where $\left(c_{x y}\right)$ is an $R_{1} \times R_{1}$ matrix with

$$
c_{x y}=1 \text { if } x=u q_{u}, y=v q_{v}, u a_{\mu v}=v, u, v \in H_{11} \text {, and } 0 \text { otherwise. }
$$

TheOREM 4. $\zeta$ is an isomorphism of $M^{\prime}\left(H_{11}, \Lambda\right)$ into $M^{\prime}\left(1, R_{1}\right)$ and $M_{D} \zeta=\Phi_{D} d$.

Proof. First observe that every element in $R_{1} \cap L_{\mu}$ can be written in the form $u q_{\mu}$ for a unique $u \in H_{11}$. Thus every element of $R_{1}$ can be uniquely written in the form $u q_{\mu}$ with $u \in H_{11}, \mu \in \Lambda$, which implies that $\zeta$ is single-valued. If $c_{x y}=c_{x z}=1$, where $x=u q_{\mu}, y=v q_{v}, z=t q_{\theta}$, then $u a_{\mu \nu}=v, u a_{\mu \theta}=t$ and hence $a_{\mu \nu} \neq 0, a_{\mu \theta} \neq 0$. But then $\nu=\theta$ since $\left(a_{\mu v}\right)$ is a row monomial matrix, which implies that $v=u a_{\mu \nu}=u a_{\mu \theta}=t$ and thus $y=z$. Therefore $\left(c_{x y}\right)$ is a row monomial matrix and $\zeta$ maps $M^{\prime}\left(H_{11}, \Lambda\right)$ into $\boldsymbol{M}^{\prime}\left(1, R_{1}\right)$.

Let $\left(a_{\mu v}\right) \zeta=\left(c_{x y}\right),\left(b_{\mu v}\right) \zeta=\left(d_{x y}\right),\left(a_{\mu v}\right)\left(b_{\mu v}\right)=\left(e_{\mu v}\right),\left(e_{\mu v}\right) \zeta=\left(f_{x y}\right),\left(c_{x y}\right)\left(d_{x y}\right)=\left(g_{x y}\right)$, $x=u q_{\mu}, y=v q_{v}, u, v \in H_{11}$. Then $f_{x y}=1 \Leftrightarrow u e_{\mu v}=v \Leftrightarrow u\left(\sum_{\theta \in \Lambda} a_{\mu \theta} b_{\theta v}\right)=v \Leftrightarrow u a_{\mu \theta} b_{\theta v}=v$ for some $\theta \in \Lambda \Leftrightarrow u a_{\mu \theta}=t, t b_{\theta v}=v$ for some $\theta \in \Lambda, t \in H_{11} \Leftrightarrow c_{x z}=d_{z y}=1$ for some $z \in R_{1} \Leftrightarrow \sum_{z \in R_{1}} c_{x z} d_{z y}=1 \Leftrightarrow g_{x y}=1$. It follows that $\left(f_{x y}\right)=\left(g_{x y}\right)$ which proves that $\zeta$ is a homomorphism. 
With the same notation suppose that $\left(c_{x y}\right)=\left(d_{x y}\right)$. Then $c_{x y}=1$ if and only if $d_{x y}=1$, whence $u a_{\mu \nu}=v$ if and only if $u b_{\mu \nu}=v$. It follows that $a_{\mu \nu} \neq 0$ if and only if $b_{\mu v} \neq 0$ and if so then $a_{\mu v}=b_{\mu v}$. Consequently $\left(a_{\mu v}\right)=\left(b_{\mu v}\right)$ and thus $\zeta$ is one-to-one.

Let $s \in S$ and let $x=u q_{\mu}, y=v q_{v}$ with $u, v \in H_{11}$. Then $s\left(M_{D} \zeta\right)=\left(a_{\mu v}\right) \zeta=\left(c_{x y}\right)$ where $a_{\mu \nu}=q_{\mu} s q_{v}^{\prime}$ if $H_{1 \mu} s=H_{1 v}$, and 0 otherwise, so that

$$
c_{x y}=1 \text { if } u q_{\mu} s q_{v}^{\prime}=v \text {, and } 0 \text { otherwise. }
$$

On the other hand, $s\left(\Phi_{D} d\right)=\beta d=\left(b_{x y}\right)$ where

$$
d \beta=\left\{x \in R_{1} \mid x s \in R_{1}\right\} \text { and } x \beta=x s \text { if } x \in d \beta,
$$

so that

$$
b_{x y}=1 \text { if } x s=y \text {, and } 0 \text { otherwise. }
$$

If $u q_{u} s=v q_{v}$, then $u q_{u} s q_{v}^{\prime}=v q_{v} q_{v}^{\prime}=v$ since $q_{v} q_{v}^{\prime}=e$ and $v \in H_{11}$. Conversely, suppose that $u q_{\mu} s q_{v}^{\prime}=v$. Then $H_{1 \mu} s=H_{1 v}$ and thus $u q_{\mu} \in H_{1 \mu}$ implies that $u q_{\mu} s \in H_{1 v}$. Consequently $u q_{\mu} s \mathscr{L} f_{v}$ (see the beginning of $\S 3$ ) with $q_{v}^{\prime} q_{v}=f_{v}$ and we have

$$
u q_{\mu} s=\left(u q_{\mu} s\right) f_{v}=u q_{\mu} s\left(q_{v}^{\prime} q_{v}\right)=v q_{v} .
$$

Using this and (7), (8), we obtain $b_{x y}=1 \Leftrightarrow x s=y \Leftrightarrow u q_{\mu} s=v q_{v} \Leftrightarrow u q_{\mu} s q_{v}^{\prime}=v \Leftrightarrow c_{x y}$ $=1$, which proves that $\left(b_{x y}\right)=\left(c_{x y}\right)$. Therefore $s\left(M_{D} \zeta\right)=s\left(\Phi_{D} d\right)$.

Roughly speaking, the effect of $\zeta$ on $\left(a_{\mu v}\right) \in M^{\prime}\left(H_{11}, \Lambda\right)$ is obtained by substituting every nonzero entry $a_{\mu \nu}$ by the $H_{11} \times H_{11}$ matrix which is its incidence matrix of the right regular representation of $H_{11}$ and every zero entry $a_{\mu \nu}$ by the $H_{11} \times H_{11}$ zero matrix and then omitting all the parentheses.

COROLlary 1. $\operatorname{ker}\left(\xi_{D} \pi_{\mathrm{P}}\right)=\operatorname{ker}\left(\chi_{D} \pi_{\mathrm{P}}\right), \operatorname{ker} \xi_{D}=\operatorname{ker} \chi_{D}$.

Proof. By the theorem, $\xi_{D} \pi_{\mathrm{P}} b d=\chi_{D} \pi_{\mathrm{P}} b d \zeta$ where $\boldsymbol{b}, \boldsymbol{d}$, and $\zeta$ are one-to-one (warning: $\pi_{\mathrm{P}}$ on the left is in general different from $\pi_{\mathrm{P}}$ on the right, the same for $\boldsymbol{b}, \boldsymbol{d})$, which proves the first part. Since $\operatorname{ker} \xi_{D}=\operatorname{ker}\left(\xi_{D} \pi_{\Lambda}\right) \cap \operatorname{ker}\left(\xi_{D} \pi_{\mathrm{P}}\right)$ and the same kind of formula holds for ker $\chi_{D}$, the first part of the corollary and its left dual imply the second assertion.

COROLlARY 2. Let $S$ be a regular semigroup and $\Delta$ be an index set of its $\mathscr{D}$-classes, then the mapping $\xi$ defined by:

$$
\xi: s \rightarrow\left(s \xi_{D}\right)_{D \in \Delta} \quad(s \in S)
$$

is an isomorphism of $S$ into $\prod_{D \in \Delta} \Omega\left(F_{D}\right)$. The mapping $\xi^{\mathrm{P}}$ defined by

$$
\xi^{\mathrm{P}}: s \rightarrow\left(s \xi_{D} \pi_{\mathrm{P}}\right) \quad(s \in S)
$$

is a homomorphism of $S$ into $\prod_{D \in \Delta} \mathrm{P}\left(F_{D}\right)$ with $\operatorname{ker} \xi^{\mathrm{P}}=\operatorname{ker} \chi^{\mathrm{P}} ; \xi^{\mathrm{P}}$ is an isomorphism if $S$ is an inverse semigroup (cf. Proposition 2).

Proof. That $\xi$ is a homomorphism is obvious. Further, using Corollary 1 we obtain

$$
\operatorname{ker} \xi=\bigcap_{D \in \Delta} \operatorname{ker} \xi_{D}=\bigcap_{D \in \Delta} \operatorname{ker} \chi_{D}=\operatorname{ker} \chi
$$


which by Proposition 2 implies that $\xi$ is one-to-one. The remaining statements follow directly from Corollary 1 and Proposition 2 .

We will use $\xi$ to embed a regular semigroup into the translational hull of a rectangular 0-band (a rectangular 0-band is a regular Rees matrix semigroup over a one element group). For this we need a new "sum" of rectangular 0-bands and two lemmas.

Lemma 2. Let $S_{\alpha}=\mathscr{M}^{0}\left(1 ; I_{\alpha}, \Lambda_{\alpha} ; P_{\alpha}\right)$ with $P_{\alpha}=\left(p_{\mu i}^{\alpha}\right), \alpha \in A$, be a disjoint family of rectangular 0-bands and suppose that $I_{\alpha} \cap I_{\beta}=\square=\Lambda_{\alpha} \cap \Lambda_{\beta}$ if $\alpha \neq \beta$. Let $I=\bigcup_{\alpha \in A} I_{\alpha}$, $\Lambda=\bigcup_{\alpha \in A} \Lambda_{\alpha}$, and define the sum $\sum_{\alpha \in A} S_{\alpha}$ by

$$
\sum_{\alpha \in A} S_{\alpha}=\mathscr{M}^{0}(1 ; I, \Lambda ; P)
$$

where $P=\left(p_{\mu i}\right)$ is a $\Lambda \times I$ matrix with

$$
p_{\mu i}=1 \text { if } \mu \in \Lambda_{\alpha}, i \in I_{\alpha} \text { for some } \alpha \in A \text { and } p_{\mu i}^{\alpha}=1 \text {, and } 0 \text { otherwise. }
$$

Then $\sum_{\alpha \in A} S_{\alpha}$ is a rectangular 0-band and is (up to an isomorphism) independent of the particular Rees matrix representation of different $S_{\alpha}$.

Proof. The regularity of $P$ follows from the regularity of each $P_{\alpha}$. Straightforward reasoning, using [3, Corollary 3.12], shows that if $S_{\alpha} \cong S_{\alpha}^{\prime}$ for all $\alpha \in S$, and $S_{\alpha}^{\prime}$ satisfy the conditions of the lemma, then $\sum_{\alpha \in A} S_{\alpha} \cong \sum_{\alpha \in A} S_{\alpha}^{\prime}$.

Lemma 3. With the notation of Lemma 2, let $S=\sum_{\alpha \in A} \oplus S_{\alpha}$ and $T=\sum_{\alpha \in A} S_{\alpha}$. Then $S$ is a subsemigroup of $T$ and $\Omega(S)$ can be embedded in $\Omega(T)$.

Proof. The first statement is obvious. Define $\delta$ by

$$
\delta:(\lambda, \rho) \rightarrow\left(\lambda^{\prime}, \rho^{\prime}\right) \quad((\lambda, \rho) \in \Omega(S))
$$

where $0 \delta=0$, and if $\rho \neq 0,0 \rho^{\prime}=0$,

$$
(1 ; i, \mu) \rho^{\prime}=(1 ; i, \mu \beta) \quad \text { if } \mu \in \Lambda_{\alpha},\left(\left.\rho\right|_{S_{\alpha}}\right) b=\beta \in W^{\prime}\left(\Lambda_{\alpha}\right), \quad \mu \in d \beta,
$$

and 0 otherwise; $\lambda^{\prime}$ is defined dually. We have seen in the proof of Lemma 1 that $\rho$ maps each $S_{\alpha}$ into itself so that indeed $\left(\left.\rho\right|_{S_{\alpha}}\right) b \in W^{\prime}\left(\Lambda_{\alpha}\right)$. One shows without difficulty that $\delta$ is an isomorphism of $\Omega(S)$ into $\Omega(T)$; we omit the details.

Let $S$ be a regular semigroup and let $\Delta$ be an index set of its $\mathscr{D}$-classes. We call $F=\sum_{D \in \Delta} F_{D}$ the fragment of $S$.

THEOREM 5. Every regular semigroup can be embedded in (i) the direct product of the translational hulls of the fragments of its different $\mathscr{D}$-classes, (ii) the translational hull of the orthogonal sum of the fragments of its different $\mathscr{D}$-classes, (iii) the translational hull of its fragment.

Proof. Part (i) is a restatement of the first statement of Corollary 2 to Theorem 4. By Lemma 1, (i) implies (ii). Finally, (ii) together with Lemma 3 implies (iii) (Lemma 2 assures that the fragment is well defined). 
The following remarks concern transitive representations by partial transformations on a set. Ponizovski [12] proved that for a large class of semigroups $S$ (including the class of finite semigroups) every transitive representation is equivalent to a representation which acts on the set $R / \rho$ where $R$ is an $\mathscr{R}$-class of $S$ contained in a nonnull principal factor and $\rho$ is a right stable equivalence relation on $R$. $\mathrm{He}$ also established when two such representations are equivalent for a smaller class of semigroups (still large enough to include all finite semigroups). If we restrict ourselves to finite semigroups $S$, then it follows easily from his results that every transitive representation of $S$ factors through $\Phi_{D}$ for some regular $\mathscr{D}$-class $D$ of $S$ (hence the name for $\Phi_{D}$ ). Furthermore, Lallement and the author [7] proved that every irreducible representation of a finite semigroup $S$ by $n \times n$ matrices over a field, roughly speaking, factors through $M_{D}$ for some regular $\mathscr{D}$-class $D$ of $S$. It follows from $M_{D} \zeta=\Phi_{D} d$ (Theorem 4) that also every transitive representation of a finite semigroup factors through $M_{D}$ for some regular $\mathscr{D}$-class $D$ of $S$. We illustrate these remarks by the following diagram. In it each loop is a commutative diagram, $\mathfrak{M}_{n}(K)$ denotes the semigroup of $n \times n$ matrices over a field $K, X$ is any nonempty set, the broken lines mean "can be completed to a commutative diagram" and concern mainly finite semigroups.

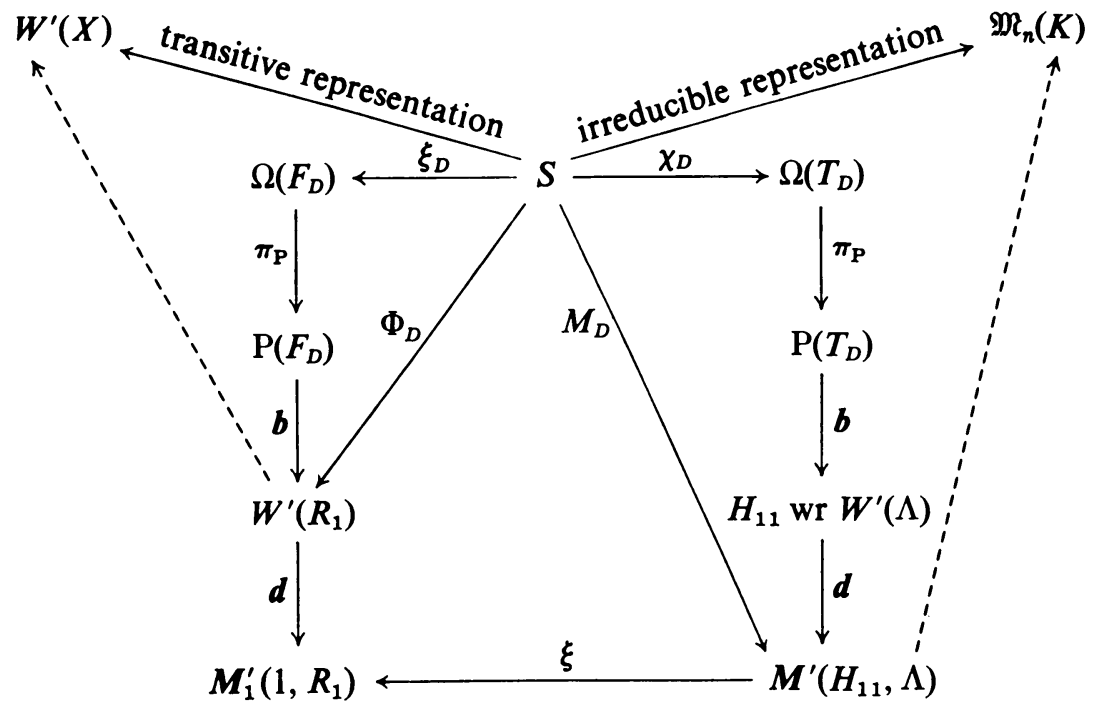

We have considered so far only regular $\mathscr{D}$-classes. If $D$ is a nonregular $\mathscr{D}$-class of a semigroup $S$, then the Schützenberger representation is a homomorphism of $S$ into $\boldsymbol{M}^{\prime}\left(\Gamma\left(H_{11}\right), \Lambda\right)$, with the usual notation. The latter semigroup is isomorphic to $\mathrm{P}\left(\mathscr{M}^{0}\left(\Gamma\left(H_{11}\right) ; I, \Lambda ; P\right)\right)$ for any regular matrix $P$ over $\Gamma\left(H_{11}\right)^{0}$ (similarly for the dual Schützenberger representation); however, there seems to be no natural candidate for $P$ ! 
5. The contour of a semigroup. Let $S$ be any semigroup and define the contour $C$ of $S$ by

$$
C=\mathscr{M}^{0}(1 ; S, S ; P)
$$

where $P=\left(p_{a b}\right)$ in an $S \times S$ matrix with $p_{a b}=1$ if $a b \in R_{a} \cap L_{b}$, and 0 otherwise. It is easy to see that $P$ is a regular matrix if and only if $S$ is a regular semigroup (use [3, Theorem 2.17]). The next theorem provides yet another embedding of a regular semigroup into the translational hull of a rectangular 0-band. For an arbitrary semigroup $S$, we define $\lambda^{s}$ and $\rho^{s}$ for $s \in S$ by:

$$
\begin{array}{ll}
\lambda^{s}(1 ; x, y)=(1 ; s x, y) & \text { if } x \mathscr{L} s x, \text { and } 0 \text { otherwise, } \\
(1 ; x, y) \rho^{s}=(1 ; x, y s) & \text { if } y \mathscr{R} y s, \text { and } 0 \text { otherwise, } \\
\lambda^{s} 0=0 \rho^{s}=0, &
\end{array}
$$

and let

$$
\eta: s \rightarrow\left(\lambda^{s}, \rho^{s}\right) \quad(s \in S) .
$$

THEOREM 6. $\eta$ is a homomorphism of $S$ into $\Omega(C)$ and

$$
\operatorname{ker}\left(\eta \pi_{\mathrm{P}}\right)=\{(s, t) \mid \text { if } x \in S \text { and either } x s \mathscr{R} x \text { or } x t \mathscr{R} x \text {, then } x s=x t\} .
$$

If $S$ is a regular semigroup, then $\operatorname{ker}\left(\eta \pi_{\mathrm{P}}\right)=\operatorname{ker} \chi^{\mathrm{P}}$ and $\eta$ is one-to-one.

Proof. The proof that $\lambda^{s}$ is a left translation and $\lambda^{s} \lambda^{t}=\lambda^{s t}$ is almost the same as the proof of the corresponding statements in Theorem 3 and is omitted; the case of right translations is dual. It remains to show that $\lambda^{s}$ and $\rho^{s}$ are linked. For any $x, y, z, w \in S$, we have

$$
(1 ; x, y)\left[\lambda^{s}(1 ; z, w)\right]=(1 ; x, y)(1 ; s z, w)=(1 ; x, w)
$$

if $z \mathscr{L}_{s z}$ and $y s z \in R_{y} \cap L_{s z}$, and

$$
\left[(1 ; x, y) \rho^{s}\right](1 ; z, w)=(1 ; x, y s)(1 ; z, w)=(1 ; x, w)
$$

if $y \mathscr{R} y s$ and $y s z \in R_{y s} \cap L_{z}$. By (9) and (10), to show that $\lambda^{s}$ and $\rho^{s}$ are linked, it remains to show that $z \mathscr{L} s z$ and $y s z \in R_{y} \cap L_{s z}$ if and only if $y \mathscr{R} y s$ and $y s z \in R_{y s} \cap L_{z}$. It is easy to see that both of these conditions are equivalent to $y s z \in R_{y} \cap L_{z}$. Therefore $\eta$ is a homomorphism of $S$ into $\Omega(C)$.

For any $s, t \in S$, we have $\rho^{s}=\rho^{t} \Leftrightarrow$ for every $(1 ; y, x) \in C,(1 ; y, x) \rho^{s}=(1 ; y, x) \rho^{t}$ $\Leftrightarrow$ for every $x \in S, x s \mathscr{R} x$ if and only if $x t \mathscr{R} x$ and if so then $x s=x t \Leftrightarrow$ if $x \in S$ and either $x s \mathscr{R} x$ or $x t \mathscr{R} x$, then $x s=x t$. This proves the second statement of the theorem. The third statement follows from Proposition 2. Taking into account that for a regular semigroup, by symmetry, we also have a corresponding statement for $\operatorname{ker}\left(\eta \pi_{\Lambda}\right)$, the last statement now follows from Proposition 2.

Definition 2. We call the representation $\Psi: S \rightarrow W^{\prime}(S)$, defined by $\Psi=\eta \pi_{\mathrm{p}} b$, the Vagner representation of $S$. Explicitly

$$
\Psi: s \rightarrow \beta^{s} \quad(s \in S)
$$

where $d \beta^{s}=\{x \in S \mid x \mathscr{R} x s\}$ and $x \beta^{s}=x s$ if $x \in d \beta^{s}$. 
This representation was proposed by Vagner [17, formula (22)], with different notation. The next proposition also follows from the theorem in [17] and [16, Theorem 6].

Proposition 5. If $S$ is an inverse semigroup, then the Vagner representation $\Psi$ of $S$ is essentially the embedding of $S$ into the symmetric inverse semigroup $V^{\prime}(S)$ due to Vagner [16], and $p_{a b}=1$ if and only if $a^{-1} a=b b^{-1}$.

Proof. First observe that $\Psi=\eta \pi_{\mathrm{P}} b$ maps $S$ into $W^{\prime}(S)$ while Vagner's embedding is a function on $S$ into $V^{\prime}(S)$, the symmetric inverse semigroup on $S$, whence "essentially" in the statement of the proposition. We have $\gamma: s \rightarrow \beta^{s}$ where $d \beta^{s}=\{x \in S \mid x \mathscr{R} x s\}$ and $x \beta^{s}=x s$ if $x \in d \beta^{s}$. It takes only little consideration to see that $d \beta^{s}=S s^{-1}$, so that the assertion follows from Theorem 1.20 [3]. Alternatively, it follows from Theorem 6 that $\Psi$ is a homomorphism, and from Theorem 6 and Proposition 2 that $\Psi$ is one-to-one. One verifies without difficulty that $\beta^{s}$ and $\beta^{\left(s^{-1}\right)}$ are mutually inverse partial transformations which proves that $\beta^{s}$ is one-toone. This establishes the first statement. For the second statement, first note that in $S, a b \mathscr{R} a$ and $a b \mathscr{L} b$ are respectively equivalent to $a=a b b^{-1}$ and $b=a^{-1} a b$. If $p_{a b}=1$, then $a b \in R_{a} \cap L_{b}$ so that $a=a b b^{-1}$ and $b=a^{-1} a b$, and thus

$$
a^{-1} a=a^{-1}\left(a b b^{-1}\right)=\left(a^{-1} a b\right) b^{-1}=b b^{-1} \text {. }
$$

Conversely, if $a^{-1} a=b b^{-1}$, then $a=a a^{-1} a=a b b^{-1}$ and $b=b b^{-1} b=a^{-1} a b$ so that $a b \in R_{a} \cap L_{b}$ and hence $p_{a b}=1$.

In comparison with Proposition 5, we have the following situation with the trace and the fragment of a regular $\mathscr{D}$-class $D$ of any semigroup. It is a simple argument to show that the statements (i) every $\mathscr{L}$ and every $\mathscr{R}$-class in $D$ contains exactly one idempotent, (ii) $T_{D}$ is a Brandt semigroup, (iii) $F_{D}$ is a Brandt semigroup, are equivalent. In such a case, according to [9, Theorem 1], the image of $\Omega\left(T_{D}\right)$ under $\pi_{\mathrm{P}} b$ is $H_{11}$ wr $V^{\prime}(\Lambda)$, and the image of $\Omega\left(F_{D}\right)$ under $\pi_{\mathrm{P}} b$ is $V^{\prime}\left(R_{1}\right)$ (with the notation used previously). Consequently $\chi_{D} \pi_{\mathrm{P}} \boldsymbol{b}$ is a homomorphism of $S$ into the inverse semigroup $H_{11}$ wr $V^{\prime}(\Lambda)$, and the Ponizovski representation $\Phi_{D}=\xi_{D} \pi_{\mathrm{P}} b$ is a homomorphism of $S$ into the symmetric inverse semigroup $V^{\prime}\left(R_{1}\right)$. In particular, a bisimple inverse semigroup $S$ can be embedded in $G$ wr $V^{\prime}(\Lambda)$ and also in $V^{\prime}\left(R_{1}\right)$ where $G$ is a maximal subgroup of $S, \Lambda$ is an index set of its $\mathscr{L}$-classes, and $R_{1}$ is any $\mathscr{R}$-class of $S$. We see that in this case the Ponizovski representation is "more economical" than the Vagner representation since the former embeds $S$ into $V^{\prime}\left(R_{1}\right)$ and the latter into $V^{\prime}(S)$. We illustrate the concepts studied in $\S \S 2,3$, and 4 with the example of a bisimple $\omega$-semigroup $S$ [13].

A bisimple $\omega$-semigroup is a bisimple inverse semigroup whose idempotents constitute a chain of the form $e_{1}>e_{2}>\cdots$. Let $N$ denote the set of nonnegative integers, $G$ be a group, and $\alpha$ be an endomorphism of $G$. On $S=N \times G \times N$ define the multiplication

$$
(m, g, n)(p, h, q)=\left(m+p-r,\left(g \alpha^{p-r}\right)\left(h \alpha^{n-r}\right), n+q-r\right)
$$


where $r=\min \{n, p\}$ and $\alpha^{0}$ is the identity mapping on $G$. Then $S$ is a bisimple $\omega$-semigroup, and conversely, every bisimple $\omega$-semigroup is isomorphic to a semigroup so constructed. Since $S$ is bisimple, $D=S$ is its only $\mathscr{D}$-class, and since it is an inverse semigroup, both $T_{D}$ and $F_{D}$ are Brandt semigroups. Let $e=e_{1}$ $=(0,1,0), r_{i}=(i, 1,0), q_{j}=(0,1, j) ;$ a simple computation shows that the following assertions are valid.

(i) $T_{D}=\mathscr{M}^{0}(G ; N, N ; \Delta)$,

$$
\begin{gathered}
d \beta^{(p, h, q)}=\{n \in N \mid n \geqq p\}, \\
n \beta^{(p, h, q)}=n+q-p, \quad n \psi^{(p, h, q)}=h \alpha^{n-p} \quad \text { if } n \in d \beta^{(p, h, q)} ;
\end{gathered}
$$

(ii) $F_{D}=\mathscr{M}^{0}(1 ; N \times G, G \times N ; \Delta)$,

$$
\begin{gathered}
d \beta^{(p, h, q)}=\{(g, n) \in G \times N \mid n \geqq p\}, \\
(g, n) \beta^{(p, h, q)}=\left(g\left(h \alpha^{n-p}\right), n+q-p\right) \quad \text { if }(g, n) \in d \beta^{(p, h, q)} ;
\end{gathered}
$$

(iii) $C=\mathscr{M}^{0}(1 ; N \times G \times N, N \times G \times N ; P)$ where

$$
\begin{gathered}
p_{(m, g, n)(p, h, q)}=1 \quad \text { if } n=p, \text { and } \frac{1}{2} \text { otherwise, } \\
d \beta^{(p, h, q)}=\{(m, q, n) \in S \mid n \geqq p\}, \\
(m, g, n) \beta^{(p, h, q)}=\left(m, g\left(h \alpha^{n-p}\right), n+q-p\right) \quad \text { if }(m, g, n) \in d \beta^{(p, h, q)} .
\end{gathered}
$$

6. A representation determined by a semilattice congruence. Let $S$ be a semigroup and $\sigma$ be a semilattice congruence on $S$ (that is, $S / \sigma$ is a semilattice). Let $Q_{\sigma}=\mathscr{M}^{0}(1 ; S, S ; P)$ where $P=\left(p_{x y}\right)$ is an $S \times S$ matrix with $p_{x y}=1$ if $x \sigma y$, and 0 otherwise. The matrix $P$ is regular since $\sigma$ is reflexive. For every $s \in S$, define $\lambda^{s}$ and $\rho^{s}$ on $Q_{\sigma}$ by:

$$
\begin{aligned}
& \lambda^{s}(1 ; x, y)=(1 ; s x, y) \quad \text { if } x \sigma s x, \text { and } 0 \text { otherwise, } \\
& (1 ; x, y) \rho^{s}=(1 ; x, y s) \text { if } y \sigma y s, \text { and } 0 \text { otherwise, } \\
& \lambda^{s} 0=0 \rho^{s}=0
\end{aligned}
$$

and let

$$
\delta_{\sigma}: s \rightarrow\left(\lambda^{s}, \rho^{s}\right) \quad(s \in S) .
$$

The proof of the assertions that follow is carried out by methods similar to those in the preceding three sections and is omitted.

THEOREM 7. $\delta_{\sigma}$ is a homomorphism of $S$ into $\Omega\left(Q_{\sigma}\right)$. Furthermore, $\delta_{\sigma}$ is one-to-one if every $\sigma$-class is weakly reductive, $\delta_{\sigma} \pi_{\mathrm{P}}$ is one-to-one if every $\sigma$-class is left reductive.

Let $\Delta_{\sigma}=\delta_{\sigma} \pi_{\mathrm{P}} b$; then $\Delta_{\sigma}: S \rightarrow W^{\prime}(S)$ is a homomorphism and is an isomorphism if every $\sigma$-class is left reductive (by Theorem 7).

PROPOSITION 6. For $\sigma$ the universal relation on $S, \Delta_{\sigma}$ is the right regular representation of $S$. For $S$ a "weakly cancellative" semigroup and $\sigma$ the maximal semilattice congruence on $S$ all of whose classes are cancellative, $\Delta_{\sigma}$ and its left dual are the 
representations introduced by Burmistrovich [2] (the proof of 2) $\Rightarrow 4$ ) in [2, Theorem 1]).

If $S$ is a regular (inverse) semigroup and $\sigma$ is a semilattice congruence on $S$, then each $\sigma$-class is a regular (inverse) semigroup (this is immediate if one observes the obvious fact that $\mathscr{D} \subseteq \sigma$ ). Hence Theorem 7 provides the embedding $\delta_{\sigma}$ of a regular semigroup $S$ into the translational hull of a rectangular 0-band, and the embedding $\Delta_{\sigma}$ of an inverse semigroup $S$ into $W^{\prime}(S)$ (for every semilattice congruence $\sigma$ on $S$ ). If $S$ is a union of groups, then $\mathscr{D}$ is a semilattice congruence, $Q_{\mathscr{D}}=C$ where $C$ is the contour of $S$, and $\delta=\eta$.

\section{REFERENCES}

1. M. I. Arbib (editor), Algebraic theory of machines, languages, and semigroups, Academic Press, New York, 1968.

2. I. E. Burmistrovich, Commutative bands of semigroups, Sibirsk. Mat. Ž. 6 (1965), 284-299. (Russian)

3. A. H. Clifford and G. B. Preston, The algebraic theory of semigroups, Vol. I, Math. Surveys No. 7, Amer. Math. Soc., Providence, R. I., 1961.

4. L. M. Gluskin, Semigroups and rings of endomorphisms of linear spaces, Izv. Akad. Nauk SSSR 23 (1959), 841-870; Amer. Math. Soc. Transl. (2) 45 (1965), 105-137.

5. — Ideals of semigroups, Mat. Sb. 55 (1961), 421-448. (Russian)

6. P. A. Grillet and Mario Petrich, Ideal extensions of semigroups, Pacific J. Math. 26 (1968), 493-508.

7. Gérard Lallement and Mario Petrich, Irreducible representations of finite semigroups, Trans. Amer. Math. Soc. 139 (1969), 393-412.

8. D. D. Miller and A. H. Clifford, Regular $\mathscr{D}$-classes in semigroups, Trans. Amer. Math. Soc. 82 (1956), 270-280.

9. Mario Petrich, The translational hull of a completely 0-simple semigroup, Glasgow Math. J. 9 (1968), 1-11.

10. - Translational hull and semigroups of binary relations, Glasgow Math. J. 9 (1968), 12-21.

11. —_ The semigroup of endomorphisms of a linear manifold, Duke Math. J. 36 (1969), $145-152$.

12. I. S. Ponizovski, Transitive representations by transformations of a class of semigroups, Sibirsk. Mat. Ž. 5 (1964), 896-903.

13. N. R. Reilly, Bisimple w-semigroups, Proc. Glasgow Math. Assoc. 7 (1966), 160-167.

14. B. M. Shain, "A contribution to the theory of generalized groups and generalized heaps" in Theory of semigroups and its applications, edited by V. V. Vagner, Izdat Saratov Univ., Saratov, 1965, pp. 286-324. (Russian)

15. E. J. Tully, Jr., Representation of a semigroup by transformations of a set, Doctoral dissertation, Tulane University, New Orleans, La., 1960.

16. V. V. Vagner, Generalized groups, Dokl. Akad. Nauk SSSR 84 (1952), 1119-1122. (Russian)

17. - Semigroups of partial transformations with the symmetric transitivity relation, Izv. Vysš. Učebn. Zaved. Matematika 1 (1957), 81-88. (Russian)

The Pennsylvania State University, University Park, Pennsylvania 